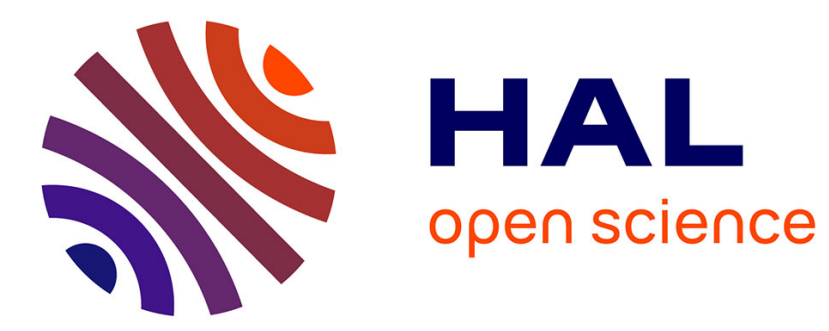

\title{
Adaptive Controller and Observer for a Magnetic Microrobot
}

\author{
Laurent Arcese, Matthieu Fruchard, Antoine Ferreira
}

\section{To cite this version:}

Laurent Arcese, Matthieu Fruchard, Antoine Ferreira. Adaptive Controller and Observer for a Magnetic Microrobot. IEEE Transactions on Robotics, 2013, 29 (4), pp.1060-1067. 10.1109/TRO.2013.2257581 . hal-00811642

\section{HAL Id: hal-00811642 \\ https://hal.science/hal-00811642}

Submitted on 25 Apr 2013

HAL is a multi-disciplinary open access archive for the deposit and dissemination of scientific research documents, whether they are published or not. The documents may come from teaching and research institutions in France or abroad, or from public or private research centers.
L'archive ouverte pluridisciplinaire HAL, est destinée au dépôt et à la diffusion de documents scientifiques de niveau recherche, publiés ou non, émanant des établissements d'enseignement et de recherche français ou étrangers, des laboratoires publics ou privés. 


\section{Adaptive Controller and Observer for a Magnetic Microrobot[1]}

\author{
Laurent Arcese, Matthieu Fruchard, Antoine Ferreira
}

\begin{abstract}
The present paper discusses the control design of a magnetically-guided microrobotic system in blood vessels to perform minimally invasive medical procedures. Such microrobots consist of a polymer binded aggregate of nanosized ferromagnetic particles and a possible payload that can be propelled by the gradient coils of a magnetic device. A fine modeling is developed and used to define an optimal trajectory which minimizes the control efforts. We then synthesize an adaptive backstepping law that ensures a Lyapunov stable and fine tracking despite modeling errors and estimates some key uncertain parameters. As the controller synthesis uses the microrobot unmeasured velocity, the design of a high gain observer is also addressed. Simulations and experiment illustrate the robustness to both noise measurement and some uncertain physiological parameters for a $250 \mu \mathrm{m}$ radius microrobot navigating in a fluidic environment.
\end{abstract}

Index Terms-Magnetic microrobot, nonlinear modeling, adaptive backstepping, high gain observer, noise and parametric uncertainties.

\section{INTRODUCTION}

Minimally invasive medical procedures are currently an active research area since related techniques can both reach remote places without surgical operation and improve targeting. Thereby, they result in lessened medical side effects and a better therapeutic efficiency and safety. Magnetically-deflected catheters [1], [2], [3] solve some weaknesses induced by conventional catether-based techniques, like the lack of manoeuvrability and resulting vascular injuries. So as to both improve accessibility to hard-to-reach body areas and reduce the risk of infection, another approach relies on untethered microrobots. Recent developments of microelectromechanical systems make it possible to fabricate such robots that can be injected intravenously to accomplish either targeted therapy or biosensing [4]. Yet it proves difficult to embed actuators sufficiently powerful to propel such systems in the cardiovascular system, especially when swimming against the blood flow. Most of swimming approaches consequently rely upon magnetic fields to wirelessly transmit power to microrobots. This proof-of-concept was first studied using electromagnets [5], [6] and superconducting magnets [7], [8]. Recently, magnetic microrobots have received a lot of attention since they are able to provide large motion forces and move in low Reynolds number fluids. Three main propulsion schemes have distinguishing physical features. First, helical and beating flagella [9] magnetic biogically inspired microrobots are promising for use in open fluids, e.g. for destroying kidney stones [10], or for ophtalmic surgery [11], [12]. Second, magnetotactic bacteria actuated thanks to embedded ferromagnetic magnetosomes has been demonstrated [13]. Finally, bead pulling is investigated using either experimental setups [10], [14], [15], [16] or magnetic resonance imaging devices [17].

L. Arcese is with CReSTIC EA 3804, University of Reims ChampagneArdenne, Moulin de la Housse, BP 1039, 51687 Reims cedex 2, France. laurent. arcese@univ-reims. fr

M. Fruchard is with the Laboratoire PRISME, University of Orleans, $63 \mathrm{Av}$ de Lattre de Tassigny, 18020, Bourges cedex, France. matthieu.fruchardeuniv-orleans.fr

A. Ferreira is with the Laboratoire PRISME, Ecole Nationale Supérieure d'Ingénieurs de Bourges, 88 Bd Lahitolle, 18000, Bourges, France. antoine.ferreira@ensi-bourges.fr

1 "(c) 20xx IEEE. Personal use of this material is permitted. Permission from IEEE must be obtained for all other users, including reprinting/ republishing this material for advertising or promotional purposes, creating new collective works for resale or redistribution to servers or lists, or reuse of any copyrighted components of this work in other works."

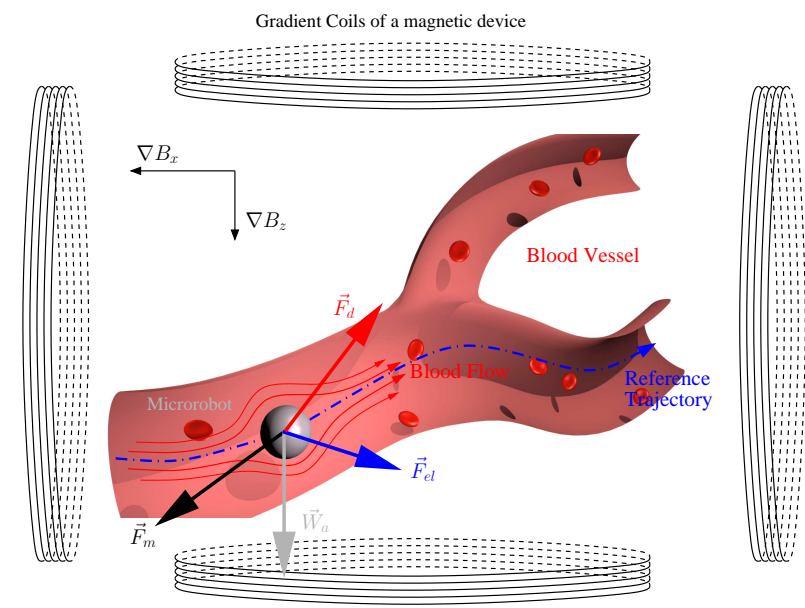

Fig. 1. Free-body diagram of forces acting on a microrobot steered by magnetic gradient fields in a blood vessel. $\vec{F}_{m}, \vec{F}_{d}, \vec{F}_{e l}$ : magnetic, hydrodynamic drag, and electrostatic forces, $\vec{W}_{a}$ : microrobot apparent weight.

All these contributions point out the difficulties of controlling magnetic microrobots in fluids through magnetic fields actuation. Achieving motion control of such robots necessitates nonlinear dynamics modeling and analysis [18], [19]. Despite nonlinearities of the proposed models, related control approaches mainly rely on linear tools of control theory. As example, the controllability of the linearized system around equilibrium is studied in [20], but there is no controller design. To deal with the nonlinearities of the magnetic field, the authors in [21] have used decoupling and linear parametrization to synthesize an optimal controller that minimizes a quadratic cost. To face the same kind of problem, a linear quadratic gaussian controller based on the linearized model has been addressed in [22]. Recently, a new approach referred to as magnetic resonance navigation has been proposed to steer and track in real-time endovascular magnetic devices in deep tissues to target areas of interest [23], [24]. As it focuses on feasibility studies of the microrobot pulling concept, developed model is linear and in turn the synthesis of control laws relies on PID approaches [24], [25]. Both authors report instabilities and important oscillations around equilibrium. These weaknesses are directly related to the use of linear controllers that are not well-suited for dealing with nonlinear perturbated systems.

In this paper, we discuss the control design of a bead pulled microrobotic nonlinear system to navigate in blood vessels. Section II recaps the nonlinear modeling of the endovascular robot and the inherited state space representation. Section III then addresses the choice of the reference trajectory, and states the robustness issues the controller has to face with. To this end, we design adaptive backstepping controllers and high gain observers in Section IV. The control design, recently developed in [26], ensures the Lyapunov stability of the trajectory tracking while high gain observers ensure the estimation error stabilization. Besides, adaptive control laws enable on-line estimation of uncertain parameters to which the system is the most sensitive, thus improving the tracking performances. Section $\mathrm{V}$ is devoted to simulation and experiment results which illustrate the stability and robustness of the controller-observer pair. Conclusions are given in the last section.

\section{BACKGROUND}

The microrobot is a polymer binded aggregate of ferromagnetic particles immersed in a small artery (Fig. 1). This section recaps the modeling of the forces that affect the microrobot, addressed in [27]. 


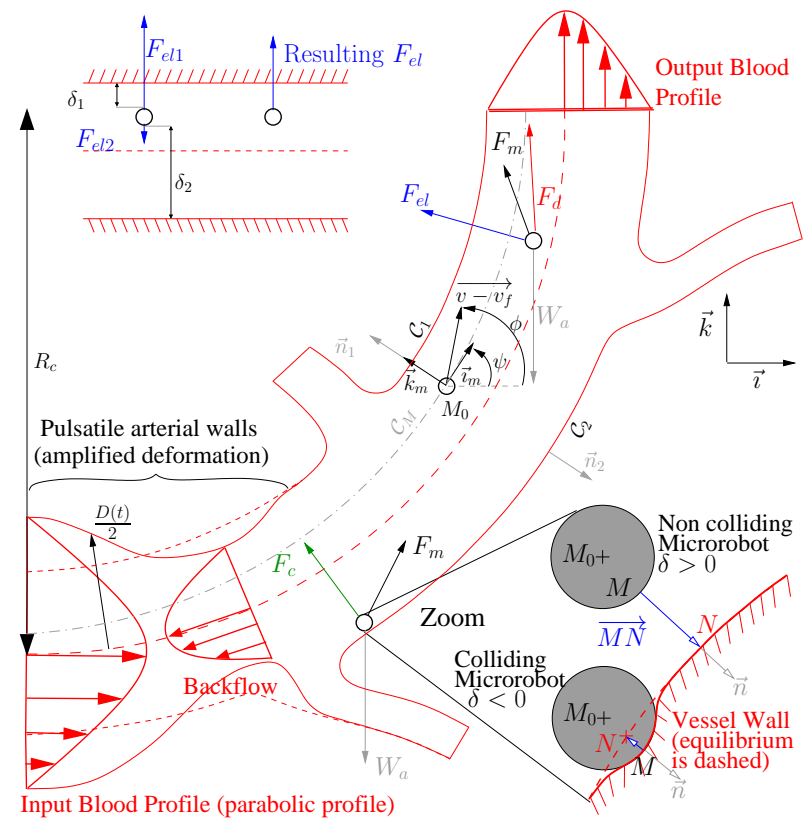

Fig. 2. Scheme of a blood vessel with minor bifurcations.

The robot translational motion is given by

$$
m \frac{d \vec{v}}{d t}=\vec{F}_{m}+\vec{F}_{d}+\vec{W}_{a}+\vec{F}_{c}+\vec{F}_{e l}
$$

where $\vec{v}$ is the translational velocity of the robot of mass $m$. In the present case study, short range interactions (van der Waals and steric forces) and stochastic effects are negligible [27].

\section{A. Hydrodynamic drag force}

The hydrodynamic drag force $\vec{F}_{d}$ exerting on a spherical body of radius $r$ in a finite fluid is expressed as:

$$
\vec{F}_{d}=-\frac{1}{2} \rho_{f}\left[\frac{\left\|\left(\vec{v}-\vec{v}_{f}\right)\right\|}{\beta}\right]^{2} A C_{d} \frac{\left(\vec{v}-\vec{v}_{f}\right)}{\left\|\left(\vec{v}-\vec{v}_{f}\right)\right\|}
$$

where $\vec{v}-\vec{v}_{f}$ denotes the relative velocity of the microrobot with respect to the fluid, $A$ is the body frontal area, $\rho_{f}$ is the blood density, $\beta$ a dimensionless ratio related to wall effect caused by the vessel occlusion by the microrobot, see e.g. [28], and $C_{d}(R e, \beta)$ is the drag coefficient [27]. The non-Newtonian behavior of blood is addressed using [29].

The wall effects result in a parabolic flow profile (see Fig. 2). The pulsative blood velocity is modeled by an affine combination of a time-varying periodic flow with the spatial shape $p(\delta) \in[0,1]$, where $\delta$ is the algebraic distance from the robot surface to the wall. In arterial vasculature, the pulsative behavior also induces a periodic deformation of the vessel diameter $D(t)$ synchronized with the pulsative blood velocity $v_{f}(t)$.

$$
v_{f}(t)=p(\delta) \sum_{n=-\infty}^{\infty} c_{n} e^{i w t}, \quad D(t)=\sum_{n=-\infty}^{\infty} d_{n} e^{i w t}
$$

\section{B. Apparent weight}

The apparent weight, that is the combined action of the weight and the buoyancy, is also acting on the microrobot:

$$
\vec{W}_{a}=V\left(\rho-\rho_{f}\right) \vec{g}
$$

where $V$ is the microrobot volume, $\rho=\tau_{m} \rho_{m}+\left(1-\tau_{m}\right) \rho_{\text {poly }}$ with $\rho_{m}$ and $\rho_{\text {poly }}$ the magnetic material and polymer densities, and $\tau_{m}=\frac{V_{m}}{V}$ the ferromagnetic ratio.

\section{Contact force}

Since the impacts occur within a few milliseconds, and under the assumption of no friction during the impact, the contact force is expressed by a modified Hertzian contact law [30] :

$$
\left\{\begin{array}{l}
\vec{F}_{c}=-K|\delta|^{3 / 2} H(-\delta) \vec{n} \quad: \text { loading } \\
\vec{F}_{c}=-F_{\delta m}\left|\frac{\delta-\delta_{0}}{\delta_{m}-\delta_{0}}\right|^{p} H(-\delta) \vec{n} \quad \text { : unloading }
\end{array}\right.
$$

where $H$ is the Heaviside step function and $\vec{n}$ the unit vector normal to the wall. $F_{\delta m}$ and $\delta_{m}$ are the maximum contact force and deformation, respectively. $\delta_{0}$ is the permanent deformation and $p \in[1.5,2.5]$. The stiffness $K$ is given in [27].

\section{Electrostatic force}

The electrostatic force between the microrobot and the wall considered as an uncharged surface is given by [31]:

$$
\vec{F}_{e l}=\frac{q^{2}}{4 \pi \epsilon \epsilon_{0}}\left(\frac{H(\delta)}{(r+\delta)^{2}}+\frac{H(-\delta)}{r^{2}}\right) \vec{n}
$$

with $q$ the microrobot charge [32] and $\epsilon$ the medium dielectric density. As depicted in Fig. 2, the resulting force includes the interactions with the upper and the lower walls.

\section{E. Magnetic force}

The gradient coils of a magnetic device provide a magnetic force $\vec{F}_{m}$ on the microrobot of magnetization $\vec{M}$ :

$$
\vec{F}_{m}=\tau_{m} V(\vec{M} \cdot \nabla) \vec{B}
$$

where $\vec{B}=B_{0} \vec{k}$ is the external magnetic field.

\section{F. State Space Representation}

Let $x_{1}, x_{2},\left(x_{3}, x_{4}\right)$ denote respectively the microrobot position and velocity along $\vec{\imath}$ axis (respectively along $\vec{k}$ axis). Assuming that positions $x_{1}$ and $x_{3}$ can be measured thanks to an imager, let $y$ denote the state measure. Using expressions of forces (2), (4), (5), (6), and (7), and adequate projections, system (1) can be written in the control-affine form

$$
\dot{x}=f(x, u)=f_{0}(x)+\sum_{i=1}^{2} f_{i}(x) u_{i}, \quad y=h(x)
$$

with vector fields given by:

$$
\left\{\begin{array}{l}
f_{0}=\left(x_{2}, f_{02}(x), x_{4}, f_{04}(x)\right)^{T}, \\
f_{1}=(0, a, 0,0)^{T}, \quad f_{2}=(0,0,0, a)^{T}
\end{array}\right.
$$

where the control inputs $\left(u_{1}, u_{2}\right)=\left(\nabla B_{x}, \nabla B_{z}\right)$ are the magnetic gradients, $a=\frac{\tau_{m} M}{\rho} \neq 0$, and the functions $f_{0 i}$ are given by :

$$
\left\{\begin{array}{l}
f_{02}(.)=F_{d n_{x}}+F_{e l n_{x}}+F_{c n_{x}} \\
f_{04}(.)=F_{d n_{z}}+F_{e l n_{z}}+F_{c n_{z}}+W_{a n}
\end{array}\right.
$$

with

$$
\left\{\begin{array}{c}
F_{d n_{x}}=\frac{3 \cos (\phi)}{2 r \beta \rho}\left[\frac{3 \eta}{r}\left\|\vec{v}-\vec{v}_{f}\right\|+\frac{\rho_{f}}{10 \beta}\left\|\vec{v}-\vec{v}_{f}\right\|^{2}\right. \\
\left.+\frac{3 \rho_{f}}{2 \beta} \frac{\left\|\vec{v}-\vec{v}_{f}\right\|^{2}}{1+\sqrt{\frac{2 \rho_{f} r}{\beta \eta}\left\|\vec{v}-\vec{v}_{f}\right\|}}\right] \\
F_{e l n_{x}}=\frac{3 q^{2} \sin (\psi)}{16 \pi^{2} r^{3} \rho \epsilon \epsilon_{0}}\left[\frac{H\left(\delta_{2}\right)}{\left(r+\delta_{2}\right)^{2}}-\frac{H\left(\delta_{1}\right)}{\left(r+\delta_{1}\right)^{2}}\right. \\
\left.\quad+\frac{H\left(-\delta_{2}\right)-H\left(-\delta_{1}\right)}{r^{2}}\right] \\
F_{c n_{x}}=\frac{3 K}{4 \pi r^{3} \rho}\left[\left|\delta_{1}\right|^{3 / 2} H\left(-\delta_{1}\right)-\left|\delta_{2}\right|^{3 / 2} H\left(-\delta_{2}\right)\right] \sin (\psi)
\end{array}\right.
$$




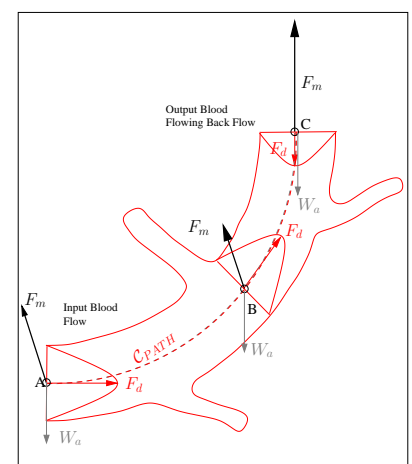

(a) Centered pre-planned path.

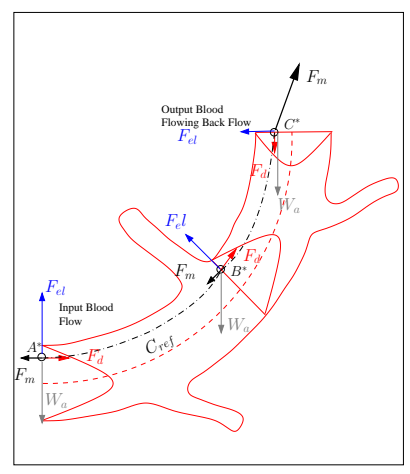

(b) Optimal trajectory.
Fig. 3. Without optimal trajectory, the control efforts $\vec{F}_{m}$ required to track the reference are beyond the capabilities of actuators. Using an optimal trajectory, external forces tend to cancel each other, resulting in a feasible tracking.

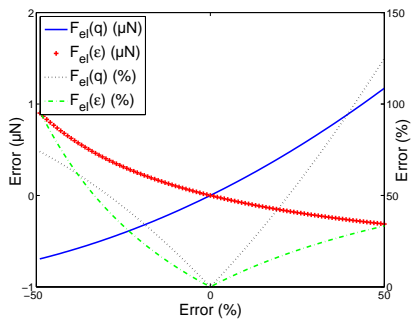

(a) Electrostatic force.

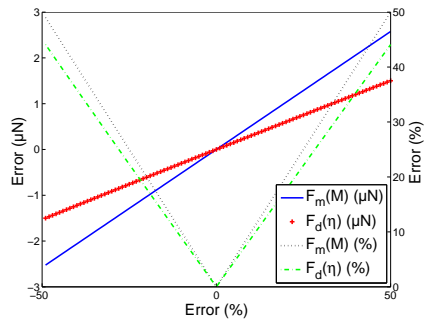

(b) Magnetic and drag forces.
Fig. 4. $\vec{F}_{m}, \vec{F}_{d}, \vec{F}_{e l}$ forces estimation error due to parametric uncertainties.

where indexes $x, z, n$ denote projections on $\vec{\imath}$ and $\vec{k}$, and normalization with respect to mass. $\psi$ and $\phi$ are used to project the mobile frame and relative velocity, respectively (Fig. 2).

\section{Problem Statement}

Prior to address system (8) control issues, we first define a reference trajectory that minimizes the control efforts. Since the robot modeling involves many physical and physiological parameters affected by uncertainties, we also provide a sensitivity analysis so as to identify the ones that deserve an on-line estimation to improve the robustness to parametric errors.

\section{A. Reference Trajectory}

An optimal trajectory is deduced from the force balance, so as to minimize the control efforts (see Fig. 3(a) and 3(b)). First, anchor points are defined in the vicinity of a pre-planned centered path. At these points, the interaction force equipoises other external forces, resulting in an optimal ratio of the motive magnetic force over external ones. A class $\mathcal{C}^{2}$ B-spline then joins these points. Along this optimal trajectory, the required control inputs are less demanding than alongside the vessel centerline, for the magnetic force should no more compete with the entirety of external forces. See [27] for more details.

\section{B. Sensitivity study}

The model (11) exhibits numerous physiological and physical parameters. A comprehensive study has been conducted to determine the predominant parameters in the model, i.e., the ones that substantially modify the estimated force in case of parametric uncertainties. This study shows how and to what extent parametric errors impacting either the electrostatic force $\vec{F}_{e l}$, the drag force $\vec{F}_{d}$, or the magnetic force $\vec{F}_{m}$, affect the estimate of the resultant force acting on the microrobot.

Figure 4 shows both the force error (in $\mu N$ ) and the force error ratio (in \%) for some parametric errors in the range $\pm 50 \%$ around their nominal values. The study is performed assuming a microrobot radius $r=250 \mu \mathrm{m}$. The center of mass of the microrobot is located at $D / 4$ from the blood vessel centerline. Figure 4(a) shows the electrostatic force error induced by uncertainties on the charge $q$ and on the medium dielectric density $\varepsilon$. The resulting nonlinear profiles are related to the fact that $\vec{F}_{e l}$ grows in $q^{2}$ and $1 / \varepsilon$, respectively. Both magnetic and drag force errors are drawn on Fig. 4(b), for parametric uncertainties on the microrobot magnetization $M$ and the fluid viscosity $\eta$, respectively. Despite the linear dependency of $\vec{F}_{m}$ in $M$, this parameter impacts severely the estimated force, since a $50 \%$ error on the robot magnetization causes a $2 \mu N$ error on the force. Although the drag force $\vec{F}_{d}$ varies as a non-linear function of $\eta$, the drag force error is quasi-linearly affected in the considered range. Both figures show that a $50 \%$ error on either $q, \varepsilon$, or $\eta$, leads to a $1 \mu N$ error on the estimation of the electrostatic and drag forces.

A significant error on one of these parameters can significantly degrade the system performances or make it unstable. Therefore, the dedicated controller should both stabilize the microrobot along a reference trajectory, and ensure robustness to the most sensitive parameters uncertainties.

\section{Main Results}

We now synthesize an adaptive backstepping control law, that Lyapunov stabilizes the system (8) along the reference trajectory and also on-line estimates some parameters in order to improve the robustness and the quality of the tracking. Since the inherited control law requires the unmeasured robot velocity, a high gain estimator is also developed. We first state on the controllability and observability of the system (8).

\section{A. Prerequisites}

Proposition 1 Let $X_{\text {ref }}=\left[X_{r}(t), \dot{X}_{r}(t), \ddot{X}_{r}(t)\right]$ denote any continuous and bounded reference trajectory. The system (8) is locally controllable along the reference trajectory.

Proof: To state a result on the controllability of system (8), we linearize it along the reference trajectory. From (8), we obtain a continuous linear time-variant system :

$$
\dot{x}=A(t) x(t)+B(t) u(t)
$$

with

$$
\left\{\begin{aligned}
A(t) & =\left.\frac{\partial f_{0}(x)}{\partial x}\right|_{X_{r e f}} \\
B(t) & =\left[f_{1} f_{2}\right] .
\end{aligned}\right.
$$

Set

$$
B_{0}=B(t), B_{i+1}=A(t) B_{i}-\frac{d B_{i}}{d t} \forall i \in \mathbb{N}
$$

The system (12) is controllable [33] along $X_{\text {ref }}$ since $\operatorname{dim}\left[B_{0} B_{1} \ldots\right]=4$ with

$$
\left[\begin{array}{ll}
B_{0} & B_{1}
\end{array}\right]=\left(\begin{array}{cccc}
0 & 0 & a & 0 \\
a & 0 & a \partial f_{02} /\left.\partial x_{2}\right|_{X_{r e f}} & a \partial f_{02} /\left.\partial x_{4}\right|_{X_{r e f}} \\
0 & 0 & 0 & a \\
0 & a & a \partial f_{04} /\left.\partial x_{2}\right|_{X_{r e f}} & a \partial f_{04} /\left.\partial x_{4}\right|_{X_{r e f}}
\end{array}\right) .
$$

Since the linearized system (12) is controllable along $X_{r e f}$, the nonlinear system (8) is locally controllable along the same trajectory [34]. 
Proposition 2 The system (8) is observable.

Proof: The observability matrix $\mathcal{O}=\left(\partial L_{f}^{i} h(x) / \partial x_{j}\right), 0 \leq$ $i \leq 1,1 \leq j \leq 4$, where $L_{f}^{i} h(x)$ denotes the $i$ th Lie derivative of the output $h(x)$ of the system (8) along the vector field $f$, is a permutation matrix, hence the result.

\section{B. Control}

The model exhibits many parameters that are unknown or highly variable. An adaptive nonlinear control law [35], [36] based on a backstepping approach is now developed to guarantee the stability in spite of parametric errors, using control Lyapunov functions (CLF). As shown in section III-B, the most sensitive parameters are $M, \eta, q$ and $\varepsilon$. However, the adaptive backstepping approach only addresses estimation of linear parameters, like $M$, - or linear up to some change of coordinates, like $q^{2} / \varepsilon$ - of the model. We here address two cases: the estimation of linear parameters in the drift vector field $f_{0}$, and in the control vector fields. We first propose the following change of variables so as to have a triangular system:

$$
X=\left(x_{1}, x_{3}\right), Z=\left(x_{2}, x_{4}\right), Y=X, u=\left(u_{1}, u_{2}\right)
$$

To deal with the unknown parameter $\theta=\frac{q^{2}}{\varepsilon}$, we set the new system from (8) and (15):

$$
\left(\mathcal{S}^{\prime}\right)\left\{\begin{array}{l}
\dot{X}=Z \\
\dot{Z}=F_{0}^{\prime}(X, Z)+\varphi_{0}(X) \theta+a u
\end{array}\right.
$$

where $F_{0}^{\prime}=\left(f_{02}-F_{e l n_{x}}, f_{04}-F_{e l n_{z}}\right)$ and $F_{e l n}=\varphi_{0}(X) \theta$.

Proposition 3 Under assumptions of Proposition 1, the adaptive backstepping control law

$$
\left\{\begin{array}{c}
u=a^{-1}\left[-\left(k_{1}+k_{2}\right)\left(Z-\dot{X}_{r}\right)-\left(1+k_{1} k_{2}\right)\left(X-X_{r}\right)\right. \\
\left.\quad-F_{0}^{\prime}(X, Z)-\varphi_{0}(X) \hat{\theta}+\ddot{X}_{r}\right] \\
\dot{\hat{\theta}}=\Gamma \varphi_{0}^{T}\left[Z-\dot{X}_{r}+k_{1}\left(X-X_{r}\right)\right]
\end{array}\right.
$$

stabilizes system (16) along any $\mathcal{C}^{0}$ reference trajectory for any initial estimate $\hat{\theta}(0)$, with $k_{1}, k_{2}>0$ the controller gains, and $\Gamma$ a positivedefinite matrix gain.

Proof: The proof is given in Appendix A.

To address robustness to an unknown linear parameter in the control vector fields, e.g. the most sensitive one, magnetization $\theta=M>0$, we set the following system from (8):

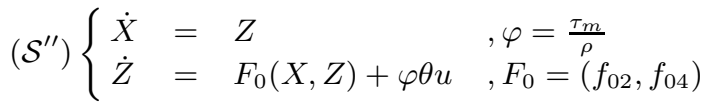

Proposition 4 Under assumptions of Proposition 1, the adaptive backstepping control law

$$
\left\{\begin{array}{ccc}
u= & (\varphi \hat{\theta})^{-1}\left[\ddot{X}_{r}-\left(k_{1}+k_{2}\right)\left(Z-\dot{X}_{r}\right)-F_{0}\right. \\
\dot{\hat{\theta}}= & \left.-\left(1+k_{1} k_{2}\right)\left(X-X_{r}\right)\right] \\
& P_{\epsilon}\left(\Gamma \varphi u^{T}\left[Z-\dot{X}_{r}+k_{1}\left(X-X_{r}\right)\right]\right)
\end{array}\right.
$$

stabilizes system (18) along any $\mathcal{C}^{0}$ reference trajectory for any initial estimate $\hat{\theta}(0) \geq \epsilon$, with $\epsilon>0$ any known lower bound on $\theta, k_{1}, k_{2}>$ 0 the controller gains, $\Gamma$ a positive-definite matrix gain, and the projection operator

$$
P_{\epsilon}\left(u_{\theta}\right)=u_{\theta} \begin{cases}0, & \text { if } \hat{\theta}=\epsilon \text { and } u_{\theta}<0 \\ 1, & \text { otherwise }\end{cases}
$$

Proof: The proof is given in Appendix B.

\section{Observer}

The control laws (17) and (19) require both the microrobot position and velocity. The former is measured by the imaging device, whereas the latter is not, which justifies the necessity of an observer. Since the vector fields are locally Lipschitz continuous, we propose high gain observers [37].

Proposition 5 Let $\mathcal{K}$ denote any compact subset of a neighborhood of $\left(X_{r}, \dot{X}_{r}\right)$, and $\mathcal{U}$ the compact set of admissible control inputs. Then $\forall(X, Z)(0) \in \mathcal{K}, \forall(\hat{X}, \hat{Z})(0) \in \mathbb{R}^{4}, \forall \hat{\theta}(0)$, there exists $L_{0}>1$ such that $\forall L>L_{0}$,

$$
\left\{\begin{array}{l}
\dot{\hat{X}}=\hat{Z}+L G_{1}(\hat{X}-Y) \\
\dot{\hat{Z}}=\widetilde{F}(\hat{X}, \hat{Z}, \hat{\theta}, u)+L^{2} G_{2}(\hat{X}-Y)
\end{array}\right.
$$

with $F=F_{0}^{\prime}+\varphi_{0} \theta+a u\left(\right.$ resp. $\left.F=F_{0}+\varphi \theta u\right)$ and $\widetilde{F}$ a globally Lipschitz extension of $F$, is a high gain observer for system (15)(16) (resp. system (15)-(18)) on $\mathcal{K}$, with $G_{1}$ and $G_{2}$ defined from the Hurwitz matrix $H_{u}$

$$
H_{u}=\left(\begin{array}{cc}
G_{1} & I_{2} \\
G_{2} & 0
\end{array}\right) \quad \text { with } \quad G_{i}=\left(\begin{array}{cc}
g_{i 1} & 0 \\
0 & g_{i 2}
\end{array}\right), i=1,2
$$

Proof: The proof is given in Appendix C.

\section{Simulations ANd ExPERIMENTAl Results}

\section{A. Simulations Settings}

Simulations are performed by taking into account the limitations of the actuators. In order not to exceed the capacity of the magnetic device, the control inputs are time-scaled as $u_{a}(t)=u(t) / k(t)$ with $k(t)=\max \left[1, u(t) / u_{\text {sat }}\right]$. Our studies assume the presence of minor bifurcations (see Figure 2). The developed controller must be sufficiently robust to compensate this effect considered as a disturbance. The velocity profile in a major bifurcation is studied in [27]. The robot navigates in a small artery of nominal diameter $D=3 \mathrm{~mm}$. The pulsatile parameters of both blood flow and arterial diameter in (3) are set to $c_{0}=1, c_{1}=c_{-1}=0.575, d_{0}=D$, $d_{1}=d_{-1}=D / 20$, and $\forall i \in \mathbb{Z} \backslash\{-1,0,1\}, c_{i}=d_{i}=0$. The performances and the stability of the controller with respect to noise measurement, parameters variations and uncertainties are now illustrated by simulations, whose nominal parameters are given in Table I.

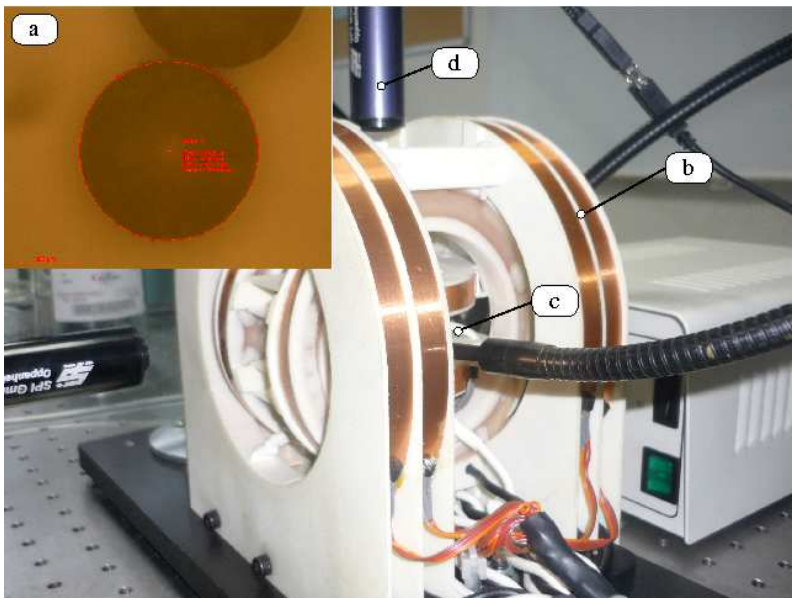

Fig. 5. Experimental setup overview: (a) $257 \mu \mathrm{m}$ radius robot, (b) magnetic actuator (three inner orthogonal Maxwell coils and one outer Helmholtz coil) from Aeon Scientific www.aeon-scientific.com, (c) workspace, (d) video microscope TIMM400. 

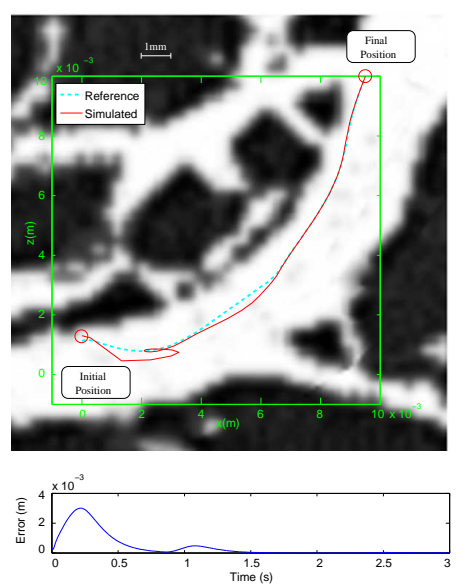

(a) Simulation 1: XY trajectory.
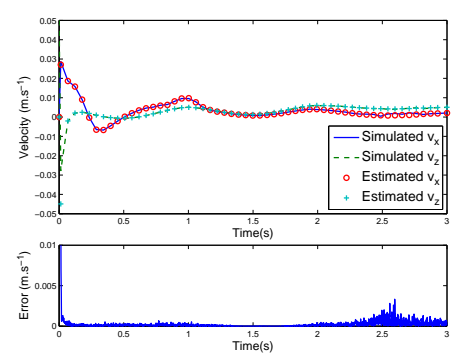

(e) Simulation 1: Observer.

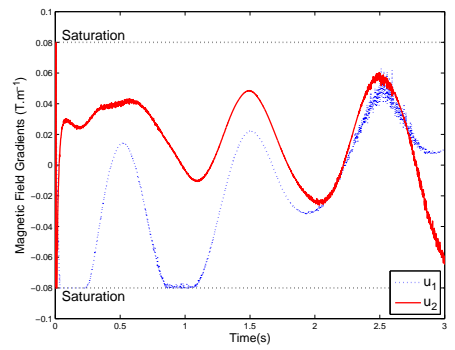

(i) Simulation 1: control input.
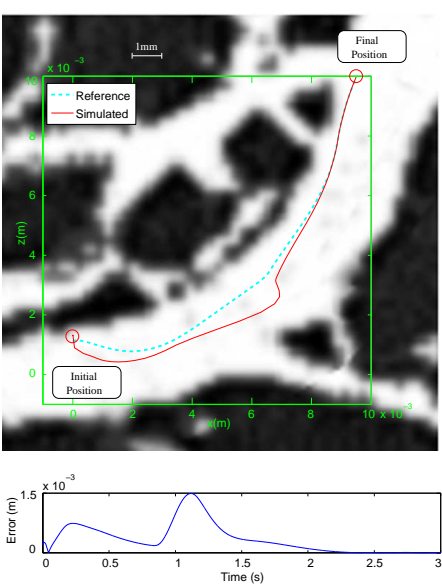

(b) Simulation 2: XY trajectory.

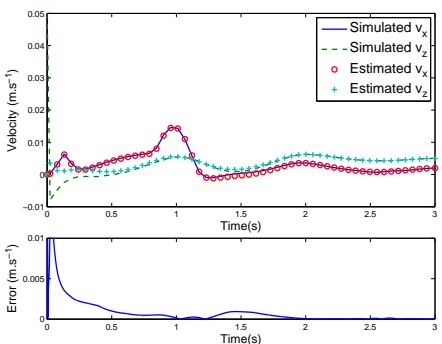

(f) Simulation 2: Observer.

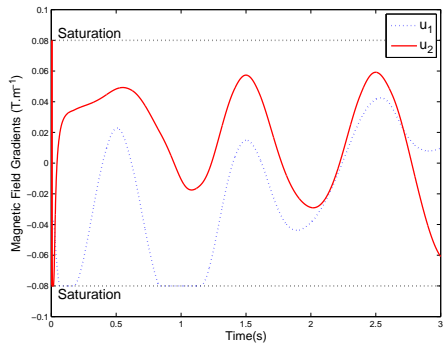

(j) Simulation 2: control input.
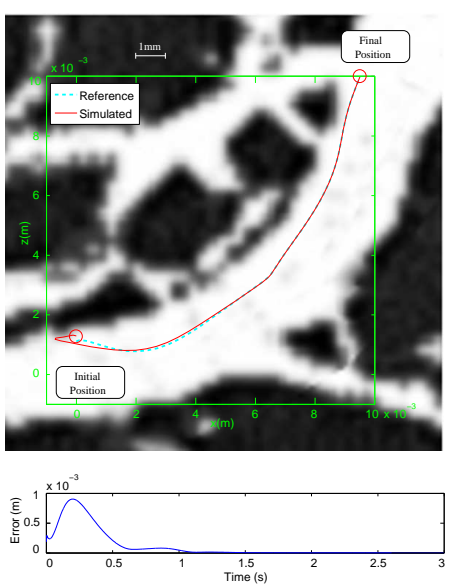

(c) Simulation 3: XY trajectory.

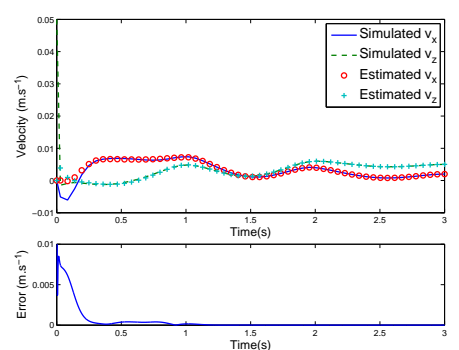

(g) Simulation 3: Observer.

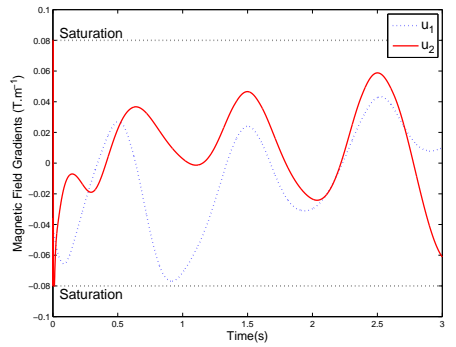

(k) Simulation 3: control input.
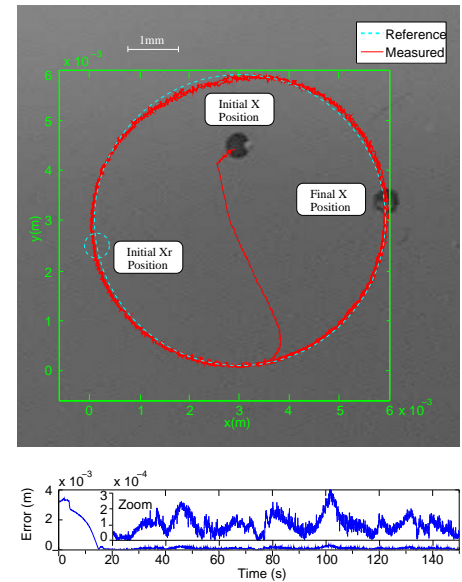

(d) Experiment: $\mathrm{XY}$ trajectory.

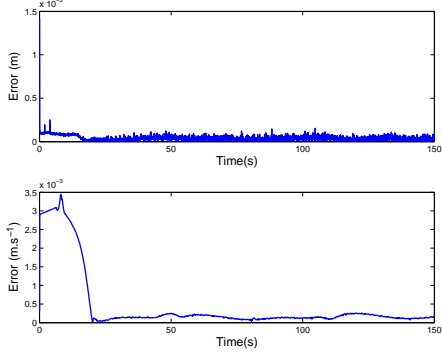

(h) Experiment: Observer.

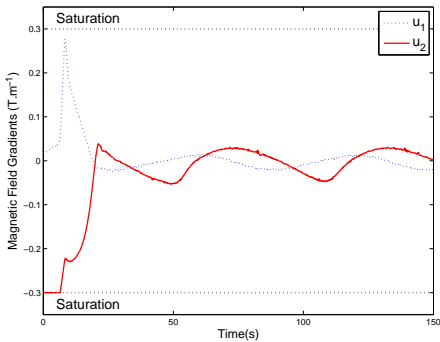

(1) Experiment: control input.

Fig. 6. From left to right: Simulation 1 (measurement noise), Simulation $2\left(20 \%\right.$ error on $F_{e l}$ ), Simulation 3 (20\% error on $F_{m}$ ), and Experimental results. (a)-(d) Reference (cyan dash line) and simulated/ real (red solid line) trajectories, and tracking norm error. (e)-(g) Simulated and estimated velocities along $\vec{\imath}$ and $\vec{k}$ axes and velocity estimation norm error. (h) Position estimation error and velocity error between reference and observer. (i)-(l) Control input : magnetic field gradients on $\vec{\imath}$-axis (blue dash line) and on $\vec{k}$-axis (red solid line).

TABLE I

SiMULATIONS DATA

\begin{tabular}{c|c|c}
\hline Blood density & $\rho_{f}$ & $1060\left[\mathrm{~kg} \cdot \mathrm{m}^{-3}\right]$ \\
\hline Magnetic material density & $\rho_{m}$ & $7500\left[\mathrm{~kg} \cdot \mathrm{m}^{-3}\right]$ \\
\hline Microrobot radius & $r$ & $250[\mu \mathrm{m}]$ \\
\hline Young's modulus & $E_{p}, E_{w}$ & $10^{9}, 10^{6}[\mathrm{~Pa}]$ \\
\hline Poisson's ratios & $\sigma_{p}, \sigma_{w}$ & $0.27,0.2$ \\
\hline Polymer density & $\rho_{\text {poly }}$ & $1500\left[\mathrm{~kg} \cdot \mathrm{m}^{-3}\right]$ \\
\hline Ferromagnetic ratio & $\tau_{m}$ & 0.8 \\
\hline Magnetization & $M$ & $1.2310^{6}\left[\mathrm{~A} \cdot \mathrm{m}^{-1}\right]$ \\
\hline Blood dielectric density & $\varepsilon$ & $70\left[\mathrm{C}^{2} \cdot \mathrm{N}^{-1} \cdot \mathrm{m}^{-2}\right]$ \\
\hline Initial conditions & $X_{0}, Z_{0}$ & $(0,0.0013)^{T},(0,0.05)^{T}$ \\
\cline { 2 - 3 } & $\tilde{X}_{0}, Z_{0}$ & $(0,0)^{T},(0,0)^{T}$ \\
\hline Inputs saturations & $u_{s a t}$ & $80\left[\mathrm{mT} \cdot \mathrm{m}^{-1}\right]$ \\
\hline Controller gains & $k_{1}, k_{2}$ & 7,14 \\
\hline Observer gains & $L$ & 5 \\
\cline { 2 - 3 } & $g_{11}, g_{21}$ & $-6,-13$ \\
& $g_{12}, g_{22}$ & $-12,-4$ \\
\hline
\end{tabular}

\section{B. Experimental Setup}

The experimental setup used to provide magnetic gradient inputs, shown on Fig. 5, has been developped by Aeon Scientific www.aeonscientific.com. It consists of one Helmholtz coil and three Maxwell coils to deliver any 3D magnetic gradient input in the workspace. In this experiment, actuators saturation is set to $u_{\text {sat }}=300 \mathrm{mT} \cdot \mathrm{m}^{-1}$. A CCD video microscope TIMM400 measures the robot position within a $40 \mu \mathrm{m}$ resolution. The robot is a $257 \mu \mathrm{m}$ radius $\mathrm{NdFeB}$ N35 ball immersed in a glycerine-water solution, with a remanent magnetization $M=0.9310^{6} \mathrm{~A} \cdot \mathrm{m}^{-1}$ and $\tau_{m}=1$. Control and imaging are real-time computed with a $50 \mathrm{~ms}$ sampling time.

\section{Results}

In the first simulation, we assume that the robot position is measured within an accuracy of $100 \mu m$-consistent with the best medical imagers resolution- modeled by a white gaussian noise on the position output. The high gain is low, since it has to fulfill 


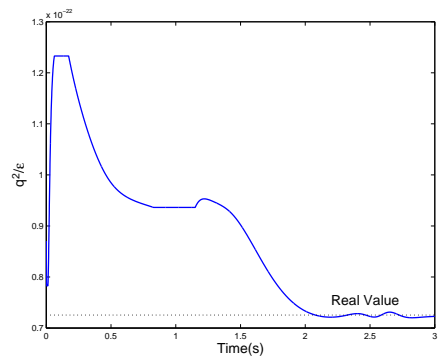

(a) Simulation 2 .

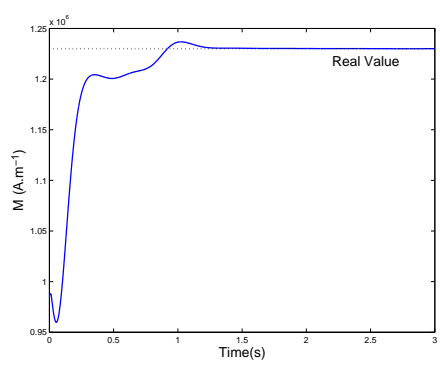

(b) Simulation 3 .
Fig. 7. On-line estimation of the unknown parameters.

a tradeoff between stability and robustness to noise. Uncertainties on the most sensitive parameters of the model are then considered: simulation 2 is performed assuming an overall $20 \%$ error on the nominal blood dielectric and maximum allowable charge, while simulation 3 shows the impact of a $20 \%$ error on the nominal magnetization value.

All simulations demonstrate the stability of the controller-observer pair. At the beginning of the first simulation (Fig. 6(a), 6(e) and 6(i)), the observer has not yet converged. Meanwhile, the microrobot collides with the blood vessel wall, forcing the control inputs to reach the saturation. This collision strongly degrades the convergence of the observer and the estimation error increases. The contact force propels the microrobot in the center of the blood vessel where the drag force is the highest. From $t=0.1 \mathrm{~s}$, the observer reconstructs correctly the state and has a filtering effect (estimation error around $30 \mu \mathrm{m}$ ). Yet, the microrobot is swept away by the blood flow and has to turn back to converge toward the reference trajectory. The control inputs reach again the saturation because of the important drag force acting on the robot at the vessel centerline. The sensitivity to noise of the control inputs around $t=0.5 \mathrm{~s}$ and $t=2.5 \mathrm{~s}$ is explained by the predominance of interaction force with respect to the hydrodynamic force (diastolic phase). Indeed, the interaction force only depends on the position measured while the hydrodynamic drag mainly depends on the velocity.

In the second and third simulations, the parametric errors affect neither the stability of the closed-loop system (Fig. 6(b) and 6(c)), nor the convergence of the observer (Fig. 6(f) and 6(g)). Nevertheless, at the beginning, the transient phase is critical since the control inputs reach the saturation (Fig. $6(\mathrm{j})$ and $6(\mathrm{k})$ ), the estimated parameters are not updated (Fig. 7(a) and 7(b)), the observers have not yet converged and the microrobot collides the wall. When the control inputs are no longer saturated, the estimated parameters are updated, and the observer converges. In the second simulation, one can notice that the tracking is degraded around $t=1 \mathrm{~s}$ for two reasons. First, the over-estimated parameter has not yet converged and the microrobot deviates toward the center of the vessel. Secondly, the hydrodynamic force acting on the microrobot is high (systolic phase) and the control inputs reach the saturation. In both simulations and after the estimated parameters have converged, the controller ensures a perfect stabilization of the microrobot along the reference trajectory.

The experiment now illustrates the robustness to both noise and parametric error. The tracking initial error with respect to the reference is very important both in position and velocity (see Fig. 6(d)). The observer error is also important since observer is initialized at the center of the circle (Fig. 6(h)). Besides, levitation is activated only after a few seconds, which explains the initial saturation on the control inputs on Fig. 6(1). These important initial errors combined with the actuator saturation induce a quite long transient phase $(15 \mathrm{~s})$. Note that the velocity estimation error on Fig. 6(h) seems to converge slowly but it is misleading artifact: since the real robot velocity is not accessible, the same goes for the velocity estimation error, and we can only plot the velocity error between the observer and the reference. The tracking is then ensured with an average error around $100 \mu m$ (see the zoom on Fig. 6(d)).

\section{DISCUSSION AND CONCLUSION}

This paper provides a preliminary study that demonstrates the proof-of-concept of an innovative method to perform medical tasks by navigating in the cardiovascular system using magnetic devices. To perform this task, a precise nonlinear model is presented for a magnetically-guided microrobot in blood vessels; this model takes into account the non-Newtonian behavior of blood, electrostatic and contact forces. An optimal trajectory is then derived from this precise model, so as to minimize the control efforts, and a sensitivity study is achieved to identify the predominant parameters. After demonstrating the controllability and observability of the system, we then synthesize a Lyapunov stabilizing control law for the nonlinear model using an adaptive backstepping control approach, coupled with a high gain observer so as to rebuild the unmeasured velocities needed to implement the controller. To validate this approach and the stability of the controller-observer pair, the first simulation illustrates the robustness to output noise. Since the modeling describes both physical and physiological forces, it is also affected by many biological parameters uncertainties. To robustify the tracking with respect to these uncertainties, an adaptive backstepping law has been developed and its relevancy and efficiency is illustrated in the two last simulations, where are considered important modeling errors on two significant parameters that affect the dynamics. Experimental results then corroborate the controller and observer efficiency and robustness.

The aim of this controller is twofold. First, it ensures the Lyapunov stability of the trajectory tracking. Second, this controller performs an on-line estimation of some key parameters. This sensitivity to matched uncertainties is a challenging issue: if biological parameters are very variable among patients, the pumping blood is also very difficult to estimate (amplitude, mean value and frequency). If some parameters can be estimated using an adaptive backstepping, it will not be as simple for parameters like the blood viscosity. In fact, drag force is a non linear function of viscosity, thus breaking classical adaptive approaches assumptions. This problem remains open.

\section{ACKNOWLEDGMENT}

This work was supported by European Union's 7th Framework Program and its research area ICT-2007.3.6 Micro/nanosystems under the project NANOMA (Nano-Actuactors and Nano-Sensors for Medical Applications).

\section{APPENDIX A \\ PROOF OF PROPOSITION 3}

Using the change of variables (15), we give a constructive proof of the Lyapunov stabilizing controller in two steps.

First let

$$
\left\{\begin{array}{l}
\tilde{X}=X-X_{r} \\
\tilde{Z}=Z-\dot{X}_{r}-\alpha
\end{array}\right.
$$

denote the position and velocity error, respectively. $\alpha$ is a stabilizing function. A CLF candidate is:

$$
V_{1}=\frac{1}{2} \tilde{X}^{T} \tilde{X}+\frac{1}{2}(\hat{\theta}-\theta)^{T} \Gamma^{-1}(\hat{\theta}-\theta) \geq 0
$$

Differentiating $V_{1}$ along (16) leads to:

$$
\dot{V}_{1}=\tilde{X}^{T}(\tilde{Z}+\alpha)+(\hat{\theta}-\theta)^{T} \Gamma^{-1} \dot{\hat{\theta}}
$$


Setting $\alpha=-k_{1} \tilde{X}$, we obtain:

$$
\dot{V}_{1}=-k_{1} \tilde{X}^{T} \tilde{X}+\tilde{X}^{T} \tilde{Z}+(\hat{\theta}-\theta)^{T} \Gamma^{-1} \dot{\hat{\theta}}
$$

The second term $\tilde{X}^{T} \tilde{Z}$ will be cancelled at the next step. One can notice from the previous expressions that:

$$
\dot{\tilde{X}}=\dot{X}-\dot{X}_{r}=\tilde{Z}+\alpha=\tilde{Z}-k_{1} \tilde{X}
$$

Second, from (A.23) and (A.27), we have:

$$
\dot{\tilde{Z}}=\underbrace{F_{0}^{\prime}+\varphi \theta+a u}_{\dot{Z}}-\ddot{X}_{r} \underbrace{+k_{1}\left(\tilde{Z}-k_{1} \tilde{X}\right)}_{-\dot{\alpha}}
$$

In this step, the CLF is given by:

$$
V_{2}=V_{1}+\frac{1}{2} \tilde{Z}^{T} \tilde{Z} \quad \geq 0
$$

The derivative of $V_{2}$ along system (16) is expressed as:

$$
\begin{aligned}
\dot{V}_{2}= & \dot{V}_{1}+\tilde{Z}^{T}\left[F_{0}^{\prime}+\varphi \theta+a u+k_{1} \tilde{Z}-k_{1}^{2} \tilde{X}-\ddot{X}_{r}\right] \\
= & -k_{1} \tilde{X}^{T} \tilde{X}+\tilde{Z}^{T}\left[\left(1-k_{1}^{2}\right) \tilde{X}+k_{1} \tilde{Z}-\ddot{X}_{r}+F_{0}\right. \\
& +\varphi \hat{\theta}+a u]+(\hat{\theta}-\theta)^{T} \Gamma^{-1}\left(\dot{\hat{\theta}}-\Gamma \varphi^{T} \tilde{Z}\right)
\end{aligned}
$$

To cancel the last term in (A.30), we set:

$$
\dot{\hat{\theta}}=\Gamma \varphi^{T} \tilde{Z}
$$

and to ensure $\dot{V}_{2}$ is negative semi-definite, we set:

$$
\left(1-k_{1}^{2}\right) \tilde{X}+k_{1} \tilde{Z}-\ddot{X}_{r}+F_{0}^{\prime}+\varphi \hat{\theta}+a u=-k_{2} \tilde{Z}
$$

Using (A.27), (A.31), (A.32), and Barbalat's lemma, we hence get the result. Note that $\hat{\theta}$ is consequently bounded.

\section{APPENDIX B}

\section{PROOF OF PROPOSITION 4}

Using the same CLF than in the previous proof, we get

$$
\left\{\begin{array}{c}
u=(\varphi \hat{\theta})^{-1}\left[\ddot{X}_{r}-\left(k_{1}+k_{2}\right)\left(Z-\dot{X}_{r}\right)-F_{0}\right. \\
\left.-\left(1+k_{1} k_{2}\right)\left(X-X_{r}\right)\right] \\
\dot{\hat{\theta}}=\Gamma \varphi u^{T}\left[Z-\dot{X}_{r}+k_{1}\left(X-X_{r}\right)\right]
\end{array}\right.
$$

Since the control law (A.33a) requires that $\hat{\theta} \neq 0$, we modify the update law $\dot{\hat{\theta}}=u_{\theta}$ to guarantee that $\hat{\theta} \geq \epsilon>0$ using the projector (20). We thus have to check that the derivative of CLF $V_{2}(t)$ given by (A.29) is still negative semi-definite when $\hat{\theta}=\epsilon$ and $u_{\theta}=\Gamma \varphi u^{T} \tilde{Z}<0$. In this case, (20) leads to a frozen update, i.e., $\dot{\hat{\theta}}=0$. Differentiating (A.29) and using (A.33a), we obtain

$$
\dot{V}_{2}=-k_{1} \tilde{X}^{T} \tilde{X}-k_{2} \tilde{Z}^{T} \tilde{Z}+(\epsilon-\theta)^{T} \Gamma^{-1}\left(-u_{\theta}\right)
$$

Since $\theta \geq \epsilon, \Gamma$ is positive definite, and $u_{\theta}<0$, the last term in (A.34) is negative, which in turn implies that $\dot{V}_{2}$ is negative semi-definite.

\section{APPENDIX C \\ ProOF OF PROPOSITION 5}

Vector fields (9)-(11) are defined using compositions of norms with Lipschitz continuous or $\mathcal{C}^{1}$ functions. Since $\mathcal{C}^{1}$ functions are locally Lipschitz and using the triangle inequality, it is not difficult to show that the drift vector field $f_{0}$ is locally Lipschitz on any compact set $\mathcal{K}$. As $u$ and $\hat{\theta}$ are bounded, $F$ is hence locally Lipschitz in the state variables on any compact set $\mathcal{K}$, and consequently admits a $k_{\mathcal{K}}$ Lipschitz extension $\widetilde{F}$ in the state variables on $\mathbb{R}^{4}$ for some $k_{\mathcal{K}} \geq 0$.

Let $e=\left(e_{x}, e_{z}\right)$ with components

$$
\left\{\begin{array}{l}
e_{x}=\frac{1}{L}(\hat{X}-X) \\
e_{z}=\frac{1}{L^{2}}(\hat{Z}-Z)
\end{array}\right.
$$

for some $L \geq 1$ whose choice is discussed later. Since by definition $\left.\widetilde{F}\right|_{\mathcal{K}}=F$, system (15)-(16) or (15)-(18) coupled with (21) gives:

$$
\dot{e}=L H_{u} e+F_{L}(\hat{X}, \hat{Z}, \hat{\theta}, u)-F_{L}(X, Z, \hat{\theta}, u),
$$

with $H_{u}$ given by (22), and mapping $F_{L}=\left(0, \frac{1}{L^{2}} \widetilde{F}\right)$. Since $H_{u}$ is Hurwitz, there exists a matrix $P$ symmetric positive definite such that:

$$
H_{u}^{t} P+P H_{u}=-I_{4}
$$

We choose a candidate Lyapunov function

$$
V(t)=e^{t} P e \leq \lambda\|e\|^{2}
$$

with $\lambda>0$ denoting the higher eigenvalue of $P$. Differentiating (A.38) and using (A.37) gives:

$$
\dot{V}=-L\|e\|^{2}+2 e^{t} P\left(F_{L}(\hat{X}, \hat{Z}, \hat{\theta}, u)-F_{L}(X, Z, \hat{\theta}, u)\right)
$$

From the $k_{\mathcal{K}}$-Lipschitz continuity of $\widetilde{F}$ in the state variables, we have

$$
\|\widetilde{F}(\hat{X}, \hat{Z}, \hat{\theta}, u)-\widetilde{F}(X, Z, \hat{\theta}, u)\| \leq k_{\mathcal{K}} L^{2}\|e\| .
$$

Using also $L \geq 1$, we can bound the Lyapunov derivative (A.39):

$$
\dot{V} \leq-\left(L-2\|P\| k_{\mathcal{K}}\right)\|e\|^{2} \leq-\left(L-2\|P\| k_{\mathcal{K}}\right) \frac{V}{\lambda}
$$

Let $L_{0}=2\|P\| k_{\mathcal{K}}$. It follows from (A.41) that, $\forall L>L_{0}$, (21) is a globally asymptotic observer of (15)-(16) or (15)-(18) on the compact $\mathcal{K}$.

\section{REFERENCES}

[1] F. Creighton, Control of magnetomotive actuators for an implanted object in brain and phantom materials. University of Virginia, 1996.

[2] D. Meeker, E. Maslen, R. Ritter, and F. Creighton, "Optimal realization of arbitrary forces in a magnetic stereotaxis system," IEEE Trans. Magn., vol. 32, no. 2, pp. 320-328, 1996.

[3] Y. Eyssa, "Apparatus and method for controlling movement of an object through a medium using a magnetic field," Patent US 6842 324, 01 11, 2005. [Online]. Available: http://es.patents.com/us-6842324.html

[4] B. J. Nelson, I. K. Kaliakatsos, and J. J. Abbott, "Microrobots for minimally invasive medecine," Annual Review of Biomedical Engineering, vol. 12, pp. 55-85, April 2010.

[5] G. T. Gillies, R. C. Ritter, W. C. Broaddus, M. S. Grady, M. A. Howard, and R. G. McNeil, "Magnetic manipulation instrumentation for medical physics research," Review of Scientific Instruments, vol. 65, no. 3, 1994.

[6] T. Honda, K. I. Arai, and K. Ishiyama, "Micro swimming mechanisms propelled by external magnetic fields," IEEE Trans. Magn., vol. 32, no. 5, 1996.

[7] E. G. Quate, K. G. Wika, M. A. Lawson, G. T. Gillies, R. C. Ritter, M. S. Grady, and M. A. Howard, "Goniometric motion controller for the superconducting coil in a magnetic stereoaxis system," IEEE Trans. Biomed. Eng., vol. 38, no. 9, pp. 899-905, 1991.

[8] S.-I. Takeda, F. Mishima, S. Fujimoto, Y. Izumi, and S. Nishijima, "Development of magnetically targeted drug delivery system using superconducting magnet," Journal of Magnetism and Magnetic Materials, 2006.

[9] R. Dreyfus, J. Beaudry, M. L. Roper, M. Fermigier, H. A. Stone, and J. Bibette, "Microscopic artificial swimmers," Nature, vol. 437, pp. 862 865, October 2005.

[10] J. Edd, S. Payen, B. Rubinsky, M. L. Stoller, and M. Sitti, "Biomimetic propulsion for a swimming surgical microrobot," Proc. IEEE IROS, pp. 2583-2588, 2003.

[11] K. B. Yelin, K. Vollmers, and B. J. Nelson, "Modeling and control of untethered biomicrorobots in a fuidic environment using electromagnetic fields," Int. J. of Robot. Research, vol. 25, no. 5-6, pp. 527-536, 2006.

[12] L. Zhang, J. J. Abbott, L. X. Dong, B. E. Kratochvil, D. J. Bell, and B. J. Nelson, "Artificial bacterial flagella: Fabrication and magnetic control," Applied Physics Letters, vol. 94, no. 6, 2009.

[13] S. Martel, M. Mohammadi, O. Felfoul, Z. Lu, and P. Pouponneau, "Flagellated magnetotactic bacteria as controlled mri-trackable propulsion and steering systems for medical nanorobots operating in the human microvasculature," Int. J. of Robot. Research, vol. 28, pp. 571-582, 2009. 
[14] J. J. Abbott, K. E. Peyer, M. C. Lagomarsino, L. Zhang, L. X. Dong, I. K. Kaliakatsos, and B. J. Nelson, "How should microrobots swim?" Int. J. of Robot. Research, vol. 28, pp. 1434-1447, 2009.

[15] H. Choi, J. Choi, S. Jeong, C. Yu, J. O. Park, and S. Park, "Twodimensional locomotion of a microrobot with a novel stationary electromagnetic actuation system," Smart Material and Structures, vol. 18, p. 115017, 2009.

[16] S. Floyd, C. Pawashe, and M. Sitti, "Two-dimensional contact and noncontact micromanipulation in liquid using untethered mobile magnetic microrobot," IEEE Trans. Robot., vol. 25, no. 6, pp. 303-308, 2009.

[17] J.-B. Mathieu, G. Beaudoin, and S. Martel, "Method of propulsion of a ferromagnetic core in the cardiovascular system through magnetic gradients generated by an mri system," IEEE Trans. Biomed. Eng., vol. 53, no. 2, pp. 292-299, 2006.

[18] J. J. Abbott, O. Ergeneman, M. P. Kummer, A. M. Hirt, and B. J. Nelson, "Modeling magnetic torque and force for controlled manipulation of soft-magnetic bodies," IEEE Trans. Robot., vol. 23, no. 6, 2007.

[19] C. Pawashe, S. Floyd, and M. Sitti, "Modeling and experimental characterization of an untethered magnetic micro-robot," Int. J. of Robot. Research, vol. 28, no. 8, pp. 1077-1094, 2009.

[20] S. Bhat, J.Guez, T. P. Kurzweg, A. Guez, and G. Friedman, "Controllability of magnetic manipulation of a few microparticles in fluid," IEEE Trans. Magn., vol. 43, pp. 2427-2429, 2007.

[21] R. Probst, J. Lin, A. Komaee, A. Nacev, Z. Cummins, and B. Shapiro, "Planar steering of a single ferrofluid drop by optimal minimum power dynamic feedback control of four electromagnets at a distance," Journal of Magnetism and Magnetic Materials, vol. 323, pp. 885-896, 2011.

[22] M. Mehrtash and M. B. Khamesee, "Design and implementation of lqg/ltr controller for a magnetic telemanipulation system-performance evaluation and energy saving," Microsystem Technology, pp. 1-9, 2011.

[23] S. Martel, J.-B. Mathieu, O. Felfoul, A. Chanu, E. Aboussouan, S. Tamaz, P. Pouponneau, H. Yahia, G. Beaudoin, G. Soulez, and M. Mankiewicz, "Automatic navigation of an untethered device in the artery of a living animal using a conventional clinical magnetic resonance imaging system," Applied Physics Letters, vol. 90(11), 2007.

[24] S. Tamaz, R. Gourdeau, A. Chanu, J.-B. Mathieu, and S. Martel, "Real-time mri-based control of a ferromagnetic core for endovascular navigation," IEEE Trans. Biomed. Eng., vol. 55, no. 7, 2008.

[25] H. Choi, J. Choi, K. Cha, L. Quin, J. Li, J. O. Park, S. Park, and B. Kim, "Position stabilization of microrobot using pressure signal in pulsating flow of blood vessel," Proc. IEEE Sensors, vol. 18, pp. 723-726, 2010.

[26] L. Arcese, M. Fruchard, F. Beyeler, A. Ferreira, and B. J. Nelson, "Adaptive backstepping and mems force sensor for an mri-guided microrobot in the vasculature," Proc. IEEE ICRA, pp. 4121-4126, 2011.

[27] L. Arcese, M. Fruchard, and A. Ferreira, "Endovascular magneticallyguided robots: navigation modeling and optimization," IEEE Trans. Biomed. Eng., vol. 59(4), pp. 977-987, 2012.

[28] R. Kehlenbeck and R. D. Felice, "Empirical relationships for the terminal settling velocity of spheres in cylindrical columns," Chemical Engineering Technology, vol. 21, pp. 303-308, 1999.

[29] A. R. Pries, T. W. Secomb, and P. Gaehtgens, "Biophysical aspects of blood flow in the microvasculature," Cardiovascular Research, vol. 32(4), pp. 654-667, 1996.

[30] I. Choi and C. Lim, "Low-velocity impact analysis of composite laminates using linearized contact law," Composite Structures, vol. 66, pp. 125-132, 2004.

[31] D. Hays, "Electrostatic adhesion of non-uniformly charged dielectric spheres," Institute of Physics Conference Series, vol. 118, pp. 223-228, 1991.

[32] — Role of Electrostatics in Adhesion, in Fundamentals of Adhesion, L.-H. Lee, Ed. Plenum Press, 1991.

[33] L. M. Silverman and H. E. Meadows, "Controllability and observability in time-variable linear systems," SIAM J. on Cont. and Opt., vol. 5, pp. 64-73, 1967.

[34] A. Isidori, Nonlinear control systems. Springer-Verlag London, 1995.

[35] I. Kanellakopoulos, P. Kokotović, and A. Morse, "A toolkit for nonlinear feedback design," Systems and Control Letters, vol. 18, pp. 83-92, 1992.

[36] M. Krstić, I. Kanellakopoulos, and P. Kokotović, "Adaptive nonlinear control without overparametrization," Systems and Control Letters, vol. 19, pp. 177-185, 1992.

[37] J. Gauthier, H. Hammouri, and S. Othman, "A simple observer for nonlinear systems. application to bioreactors." IEEE Trans. Autom. Control., vol. 37, no. 6, pp. 875-880, 1992. 